\title{
Compósitos de Borracha Natural com Polianilina
}

\author{
Patrini D. Galiani, José A. Malmonge, Daniela P. dos Santos, Luiz F. Malmonge \\ Departamento de Física e Química, UNESP
}

Resumo: Compósitos de borracha natural (Hevea brasiliensis)-BN/polianilina - PANI, com diferentes composições foram obtidos através da polimerização por emulsão do monômero anilina na presença da BN e do ácido dodecilbenzeno sulfônico (DBSA). Filmes finos e homogêneos foram obtidos por prensagem a quente. Os compósitos foram caracterizados por condutividade elétrica, FTIR, UV-vis-NIR, DSC e difração de raios X. Compósito com condutividade elétrica cerca de 14 ordens de grandeza maior que a $\mathrm{BN}$ foi obtido. Este alto valor de condutividade é atribuído à formação da PANI no estado dopado no compósito, que foi verificado através das técnicas de UV-vis-NIR e FTIR. Os resultados obtidos com a técnica de DSC e difratometria de raios X indicaram que os polímeros são imiscíveis e que a presença da borracha não altera significantemente a fase cristalina da PANI-DBSA no compósito.

Palavras-chave: Polimerização por emulsão, borracha natural, polianilina.

\section{Composites of Natural Rubber with Polyaniline}

Abstract: In this work composites with different compositions were obtained from natural rubber (Hevea brasiliensis) (NR) and polyaniline (PANI) using the emulsion polymerization of aniline in the presence of NR and dodecylbenzenesulfonic acid (DBSA). The samples in film form were obtained by pressing the precipitate at $100{ }^{\circ} \mathrm{C}$ for 5 minutes. The composites were characterized by electrical conductivity, Fourier-transform infrared spectroscopy (FTIR), UV-vis-NIR spectroscopy, differential scanning calorimetry (DSC) and X ray diffraction. Composites with electrical conductivity about 14 orders of magnitude higher than NR were obtained. The UV-vis-NIR and FTIR spectra showed that PANI-DBSA was formed in the composites, thus making it responsible for their high conductivity. The DSC thermograms indicated that the two polymers are immiscible and $\mathrm{X}$ ray diffraction evidenced that NB does not considerably affect PANI-DBSA crystalline phase in the composite.

Keywords: Emulsion polymerization, natural rubber, polyaniline.

\section{Introdução}

Os polímeros intrinsecamente condutores possuem grande potencial para aplicações tecnológicas, devido principalmente a suas propriedades elétricas e óticas. Dentre esses polímeros condutores, destaca-se a polianilina-PANI devido a sua estabilidade química no estado dopado em condições ambientais e também por ser facilmente sintetizada quimicamente ${ }^{[1]}$. Entretanto, uma das limitações no uso da polianilina tem sido sua processabilidade apresentando baixa solubilidade e infusibilidade. Dentro deste contexto, blendas e compósitos com polímeros convencionais têm atraído muita atenção, uma vez que estes possibilitam a combinação das excelentes propriedades mecânicas e a alta processabilidade, com a condutividade elétrica da polianilina, aumentando o potencial tecnológico destes materiais. A polimerização via emulsão da anilina na presença de um outro polímero em solução ${ }^{[2-5]}$, tem sido uma alternativa viável. A vantagem deste método é que ele fornece uma grande quantidade de material que pode ser processado posteriormente em forma de filmes, peças etc., que muitas vezes não é possível com compósitos obtidos por outros métodos como por exemplo casting.

Dentre os polímeros convencionais, destaca-se a borracha natural que por ser oriunda de uma fonte renovável, desperta grande interesse na procura de novas aplicações. Do consumo mundial de borracha, cerca de $40 \%$ é natural e $60 \%$ sintética sendo que o látex da seringueira (Hevea brasiliensis) é o único explorado comercialmente ${ }^{[6]}$.

A borracha natural extraída do látex da seringueira é um polímero linear composto de cadeias cis-1,4-poli-isopreno de alto peso molecular ${ }^{[7]}$. No que se refere às propriedades elétricas, a borracha natural é classificada como isolante elétrico.

Encontra-se na literatura vários trabalhos onde se descreve a obtenção de compósitos de PANI com elastomeros, utilizando diferentes métodos de preparo como mistura termomecânica $^{[8,9]}$, "casting"'[10-13], eletroquímico e emulsão ${ }^{[14]}$. Neste trabalho, compósitos de borracha natural com polianilina foram obtidos através da polimerização da anilina por emulsão na presença de borracha natural, toluol e do ácido dodecilbenzeno sulfônico - DBSA. As caracterizações dos compósitos foram feitas através de medidas de Condutividade Elétrica, 
Espectroscopia UV-vis-NIR e FTIR, Difratrometria por raios X e Calorimetria Exploratória Diferencial - DSC. A borracha natural foi escolhida por ter boa propriedade mecânica, ser de baixo custo e obtida de fonte renovável. Realçamos que vários compósitos de polianilina com polímeros isolantes são obtidos utilizando este método (emulsão) mas, segundo nosso conhecimento, com borracha natural este é o primeiro trabalho.

\section{Experimental}

\section{Materiais e métodos}

A anilina (Aldrich, 99,5\%) foi purificada pela destilação a vácuo antes do uso. Os reagentes persulfato de amônio (Merck P.A), $\mathrm{HCl}$ (Synth P.A.), Metanol (Synth P.A.), Toluol (Synth P.A.), $\mathrm{NH}_{3}$ (Merck P.A.), NMP (Vetec P.A.) e o sal lauril sulfato de sódio (Aldrich P.A.) foram utilizados conforme recebidos. O ácido dodecilbenzeno sulfônico (DBSA) foi preparado no laboratório por meio da troca iônica do íon $\mathrm{Na}^{+}$pelo $\mathrm{H}^{+}$e para tal utilizou-se a resina catiônica AMBERLITE IR - 120 da Synth e o sal DBSA (Aldrich, 95\%).

\section{Borracha natural}

O látex, extraído através do processo de sangria, utilizado para obtenção da borracha natural, foi coletado de diferentes árvores de Seringueira (Hevea brasiliensis) clone RRIM - 600 localizadas na Fazenda Experimental da Faculdade de Engenharia de Ilha Solteira (FEIS/UNESP). Esse látex foi estabilizado pela adição de amônia e armazenado a uma temperatura de aproximadamente $5{ }^{\circ} \mathrm{C}$.

A purificação do látex foi feita através de sua mistura com uma solução aquosa a 5,5\% do sal lauril sulfato de sódio e então centrifugado a $12.500 \mathrm{rpm}$ por 50 minutos a $10{ }^{\circ} \mathrm{C}$. Nestas condições é possível descartar grande parte dos constituintes não borracha presentes no látex. A borracha foi obtida da coagulação, com acetona, do látex purificado. Para retirada do excesso de sal, a borracha foi lavada com água e posteriormente seca à temperatura de $60^{\circ} \mathrm{C}$ por 48 horas.

\section{Síntese da PANI-DBSA}

A PANI-DBSA foi obtida pela polimerização por emulsão da anilina de acordo com a literatura ${ }^{[2,15]}$. Foi preparada uma solução de 24,5 g de DBSA e 4,7 mL de anilina em $250 \mathrm{~mL}$ de toluol a qual foi mantida sob agitação e a uma temperatura entre $0-2{ }^{\circ} \mathrm{C}$. A esta solução acrescentou-se lentamente 4,68 $\mathrm{g}$ do oxidante persulfato de amônio dissolvido em $20 \mathrm{~mL}$ de água destilada. Após 24 horas de polimerização sob agitação e a temperatura entre $0-2{ }^{\circ} \mathrm{C}$, acrescentou-se 500 $\mathrm{mL}$ de metanol para precipitação da PANI. O precipitado obtido foi filtrado e lavado com metanol e deixado sob vácuo dinâmico à temperatura ambiente por 12 horas. A polianilina assim obtida (PANI-DBSA) apresenta-se na forma de pó no estado dopado (sal de esmeraldina) e sua estrutura química pode ser representada como:

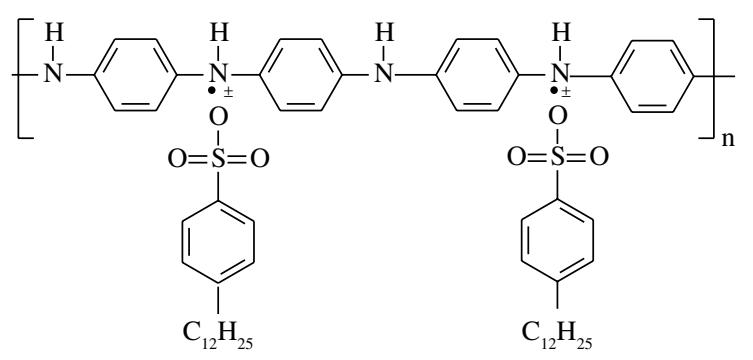

A desdopagem foi feita misturando a PANI-DBSA em uma solução aquosa de hidróxido de amônio $\left(0,1\right.$ mol.L $\left.\mathrm{L}^{-1}\right)$ por 16 horas sob agitação mecânica. A solução foi filtrada e lavada com hidróxido de amônio 0,1 mol.L ${ }^{-1}$ e deixada sob vácuo dinâmico à temperatura ambiente por 12 horas. A PANI na forma não dopada (base de esmeraldina) assim obtida, pode ser representada como segue:<smiles>CC1C=CC(=Nc2ccc(N=C3C=CC(=NC4C=CC(C)(C)CC4)C=C3)cc2)CC1</smiles>

\section{Preparação dos compósitos BN/PANI-DBSA}

Os compósitos foram obtidos através da polimerização da anilina via emulsão na presença da borracha natural. A anilina foi adicionada numa solução, sob agitação, de borracha dissolvida em toluol (5\%) misturada com o ácido DBSA, que além de dopar a PANI este age como surfactante. Em seguida foi adicionado uma solução aquosa do oxidante persulfato de amônio para iniciar a polimerização. Esta solução final foi mantida sob agitação mecânica a uma temperatura entre 0 e $2{ }^{\circ} \mathrm{C}$ por 24 horas. Após este tempo de polimerização, a emulsão foi desestabilizada adicionando acetona na solução. O precipitado obtido foi então filtrado e lavado com acetona e posteriormente seco sob vácuo a temperatura ambiente por 6 horas. As condições de polimerização utilizadas foram: razões molares $\left(\mathrm{NH}_{4}\right)_{2} \mathrm{~S}_{2} \mathrm{O}_{8}$ /Anilina $=1$ e DBSA/Anilina = 3; razão: água/toluol (v/v) = 4 e razão BN/Anilina $(\mathrm{g} / \mathrm{g})=1,5$; 3,0 e 4,0 .

Os filmes dos compósitos foram obtidos por prensagem a quente (do precipitado) a uma temperatura de $100{ }^{\circ} \mathrm{C}$ por 5 minutos.

\section{Caracterização dos compósitos}

As medidas de condutividade elétrica dos compósitos e da borracha foram feitas pelo método de quatro e duas pontas, respectivamente. No método de quatro pontas, a amostra foi mantida sob pressão em sua superfície por quatro contatos elétricos (pontas) eqüidistantes. A medida foi feita aplicando uma corrente elétrica nos contatos das extremidades e a diferença de potencial medida entre os contatos elétricos internos, sendo a condutividade elétrica calculada pela equação ${ }^{[16,17]}$

$$
\sigma=\frac{I}{V L C}
$$


onde $\sigma$ é a condutividade elétrica $\left(\mathrm{S} . \mathrm{cm}^{-1}\right), I$ a corrente aplicada, $V$ a diferença de potencial, $L$ a espessura da amostra e $C$ uma constante calculada em função da largura, comprimento e espessura da amostra e da distância entre os contatos elétri$\cos ^{[16]}$. Nas nossas medidas a constante utilizada foi $\pi / \ln 2$.

No método de duas pontas a amostra foi metalizada com alumínio, por evaporação, em ambas as faces. A condutividade elétrica foi determinada utilizando a equação ${ }^{[17]}$ :

$\sigma=\frac{I_{0} L}{\Delta \mathrm{V}}$

onde $A$ é área metalizada, $L$ a espessura da amostra, $V$ a diferença de potencial aplicada sobre a amostra e $I_{0}$ a corrente ôhmica da amostra.

Para as medidas de absorção no UV-vis-NIR utilizou-se um espectrofotômetro VARIAN CARY modelo 50 na faixa de 1100 a $300 \mathrm{~nm}$. As análises foram feitas em soluções de toluol e clorofórmio para os compósitos e clorofórmio para a PANI-DBSA. Os espectros de FTIR foram obtidos usando um espectrofotômetro NEXUS 670, Nicolet Instrument Corporation na faixa de 4000 a $400 \mathrm{~cm}^{-1}$. As amostras foram analisadas na forma de filmes, para os compósitos, e pó em pastilha de $\mathrm{KBr}$ (proporção 200/1) para a PANI-DBSA. Análises de DSC foram feitas em um sistema da TA Instruments modelo MDSC 2920 na faixa de temperatura de -100 a $200{ }^{\circ} \mathrm{C}$, com uma taxa de aquecimento de $10^{\circ} \mathrm{C} / \mathrm{min}$., em atmosfera dinâmica de nitrogênio. Os difratogramas de raios $\mathrm{X}$ foram obtidos em um difratômetro de marca RIGAKU-ROTOFLEX modelo RV-200B, com comprimento de onda característico do $\mathrm{Cu}, \mathrm{K} \alpha$ igual a 1,54 Å e com ângulo de varredura $(2 \theta)$ de 5 a $35^{\circ}$. As análises foram feitas em amostras na forma de filmes, para a BN e o compósito, e na forma de pastilha para a PANI-DBSA.

\section{Resultados e Discussão}

Os valores de condutividade elétrica medidos pelo método de quatro pontas para os compósitos e para a PANI-DBSA (na forma de pastilha), estão apresentados na Tabela 1. Verifica-se um aumento da condutividade elétrica do compósito à medida que a razão BN/Anilina diminui, alcançando um valor da ordem de $10^{-1} \mathrm{~S} / \mathrm{cm}$ para a razão 1,5 . Este valor é cerca de 14 ordens de grandeza maior do que o da borracha pura e uma ordem de grandeza inferior ao da PANI-DBSA. Observou-se que o ganho na condutividade é acompanhado da perda da propriedade elástica da borracha, observação esta feita manualmente.
Os espectros de FTIR da BN, PANI-DBSA e do compósito BN/PANI-DBSA são mostrados na Figura 1 e as principais linhas de absorção estão apresentadas na Tabela 2. Estes resultados estão de acordo com os encontrados na literatu$\mathrm{ra}^{[18-23]}$. Observa-se que as principais linhas da PANI-DBSA estão presentes no compósito comprovando a ocorrência da polimerização da PANI.

A Figura 2 ilustra os espectros de absorção de UV-visNIR da PANI-DBSA dopada e desdopada. O espectro da PANI-DBSA dopada apresentou duas bandas e um ombro de absorção. A primeira banda em $340 \mathrm{~nm}$ é atribuída à transição $\pi-\pi^{*}$ dos anéis benzenóides, o ombro em $430 \mathrm{~nm}$ e a segunda banda em $795 \mathrm{~nm}$, são devidos à presença de po-

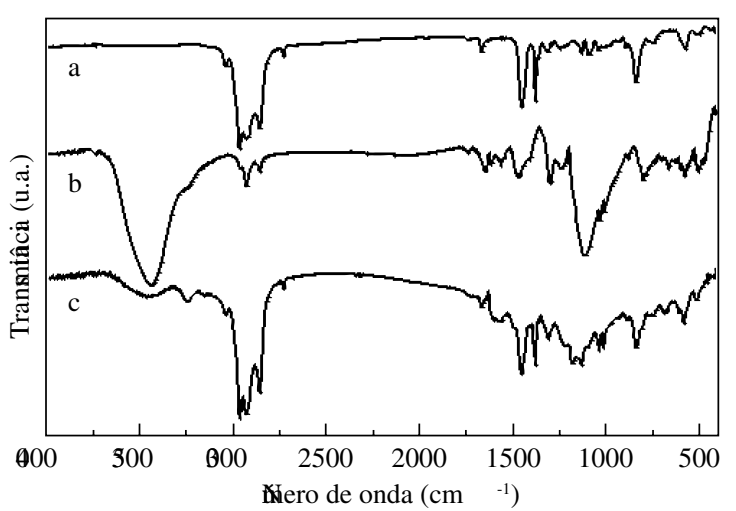

Figura 1. Espectros de FTIR: a) BN; b) PANI-DBSA; e c) do compósito BN/PANI-DBSA.

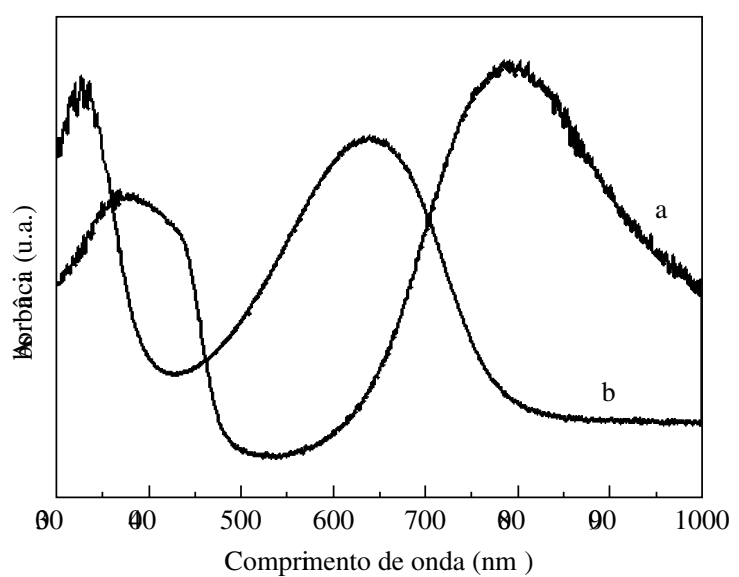

Figura 2. Espectros de UV-vis-NIR da PANI-DBSA, no estado a) dopado; e b) desdopado.

Tabela 1. Valores de condutividade elétrica da BN, PANI-DBSA e dos compósitos BN/PANI-DBSA.

\begin{tabular}{cccc}
\hline Amostras & Razão BN/anilina & Condutividade $\left(\mathbf{S . c m}^{-1}\right)$ & Desvio padrão $\left.(\mathbf{S . c m})^{-1}\right)$ \\
\hline B1 & 1,5 & $5,6 \times 10^{-1}$ & $2,1 \times 10^{-2}$ \\
B2 & 3,0 & $1,8 \times 10^{-3}$ & $5,7 \times 10^{-4}$ \\
B3 & 4,0 & $7,3 \times 10^{-4}$ & $7,8 \times 10^{-5}$ \\
PANI-DBSA & - & 1,6 & - \\
BN & - & $10^{-15}$ & - \\
\hline
\end{tabular}


Tabela 2. Principais linhas de absorção na região do infra vermelho (FTIR) da PANI-DBSA; da borracha natural (BN); e do compósito.

\begin{tabular}{|c|c|c|c|}
\hline \multirow[b]{2}{*}{ Atribuições } & \multicolumn{3}{|c|}{$\mathrm{N}^{0}$ de onda $\left(\mathrm{cm}^{-1}\right)$} \\
\hline & PANI-DBSA & Compósito & $\mathbf{B N}$ \\
\hline Deformação angular $\mathrm{C}=\mathrm{C}-\mathrm{H}^{(22)}$ & & 833 & 835 \\
\hline Estiramento $\mathrm{S}=\mathrm{O}^{(18,20,21)}$ & 1033 e 1004 & 1033 e 1008 & \\
\hline Deformação angular C-H da estrutura & 1120 & 1128 & \\
\hline $\mathrm{N}=\mathrm{Q}=\mathrm{N}$ e $\mathrm{B}-\mathrm{N}^{+} \mathrm{H}-\mathrm{B}^{(18)}$ & & & \\
\hline Estiramento da ligação C-N da amina secundária ${ }^{(18)}$ & 1297 & 1303 & \\
\hline Estiramento $\mathrm{C}=\mathrm{C}$ do anel quinóide ${ }^{(19)}$ & 1559 & 1565 & \\
\hline Deformação angular do $\mathrm{CH}_{2}^{(23)}$ & & 1448 & 1450 \\
\hline Deformação angular simétrica do $\mathrm{CH}_{3}^{(23)}$ & & 1375 & 1375 \\
\hline Deformação axial simétrico da ligação $C=C^{(23)}$ & & 1662 & 1664 \\
\hline Deformação axial assimétrica da C-H no $\mathrm{CH}_{3}^{(23)}$ & & 2960 & 2962 \\
\hline Deformação axial simétrico da ligação $=\mathrm{C}-\mathrm{H}^{(23)}$ & & 3035 & 3035 \\
\hline Estiramento $\mathrm{O}-\mathrm{H}^{(19)}$ & 3444 & 3446 & \\
\hline
\end{tabular}

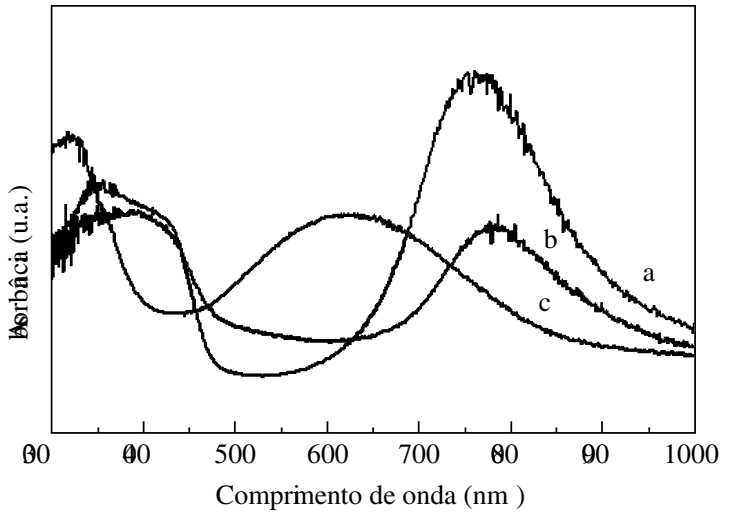

Figura 3. Espectros de absorção na região UV-vis-NIR do compósito BN/ PANI-DBSA: a) em solução de toluol; b) em solução de clorofórmio; e c) na forma de filme e desdopado em solução $\mathrm{NH}_{4} \mathrm{OH} 0,1$ mol.L $\mathrm{L}^{-1}$.

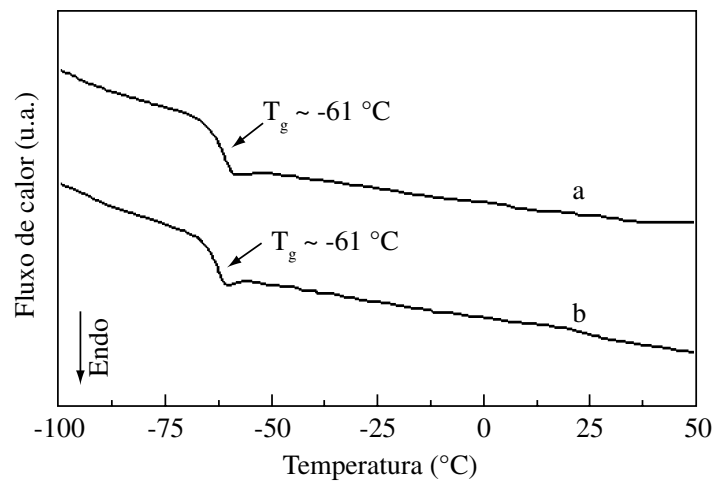

Figura 4. Termogramas de DSC: a) borracha natural; e b) filme do compósito B2 (razão BN/Anilina $=3,0$ ). Resultados similares foram obtidos para os compósitos com diferentes composições

laron formado pela protonação ${ }^{[18,24-26]}$. Para a PANI-DBSA desdopada duas bandas de absorção são observadas, uma em $325 \mathrm{~nm}$ atribuída à transição $\pi-\pi^{*}$ dos anéis benzenóides e a segunda em $634 \mathrm{~nm}$ atribuída à transição do exciton molecular que está relacionada com a transferência de cargas dos anéis benzenóides para os quinóides ${ }^{[26,27]}$.

Os espectros de absorção UV-vis-NIR do compósito BN/PANI-DBSA dopado e desdopado são apresentados na
Figura 3. Verifica-se nos espectros as mesmas transições eletrônicas observadas para a PANI-DBSA, indicando a formação de polianilina no compósito, como também verificado nas análises de FTIR. A polianilina dopada no compósito é a responsável pelo alto valor da condutividade conferida ao mesmo.

Pode-se observar que a segunda banda $(765 \mathrm{~nm})$ do espectro do compósito (Figura 3b,c) apresenta um deslocamento em relação a PANI-DBSA(Figura 2a). Esse deslocamento está associado à mudança de conformação das cadeias da polianilina, ficando mais enrolada (coil) no compósito. Esta conformação é influenciada por diversos fatores tais como ${ }^{[26,28]}$ : do solvente usado para preparar a amostra, do dopante, da matriz polimérica na blenda e da razão molar dopante/polianilina. No nosso caso, esta mudança de conformação foi influenciada fortemente pelo solvente, uma vez que o espectro do compósito dissolvido em clorofórmio deslocou para maior comprimento de onda como mostra a curva b da Figura 3.

O comportamento da transição vítrea $\left(\mathrm{T}_{\mathrm{g}}\right)$ nos compósitos foi verificado através da técnica de DSC. Na Figura 4 encontram-se os termogramas de DSC para a borracha pura e para o compósito B2 (razão BN/Anilina $(\mathrm{g} / \mathrm{g})=3,0$ ). Observa-se que não houve variação na temperatura de transição vítrea. Este resultado sugere que os polímeros são imiscíveis.

A Figura 5 mostra os difratogramas de raios $\mathrm{X}$ da $\mathrm{BN}$, do compósito B2 e da PANI-DBSA. O difratograma da BN apresenta característica de um polímero amorfo, com uma banda de espalhamento centrado, em $2 \theta \sim 18,5^{\circ}$. O difratograma da PANIDBSA é típico da PANI na forma de pó sintetizada quimicamente $^{[18,19]}$. Os picos $2 \theta=5,2 ; 7,8 ; 18,8$ e $25,2^{\circ}$ correspondem no plano hkl (200), (300), (001) e (110), respectivamente. Para o compósito B2 picos referentes a PANI-DBSA são observados indicando que a borracha não altera significativamente a fase cristalina da PANI-DBSA, com exceção do pico em $2 \theta=25,2^{\circ}$ que aparece com baixa intensidade proporcionalmente aos demais. 


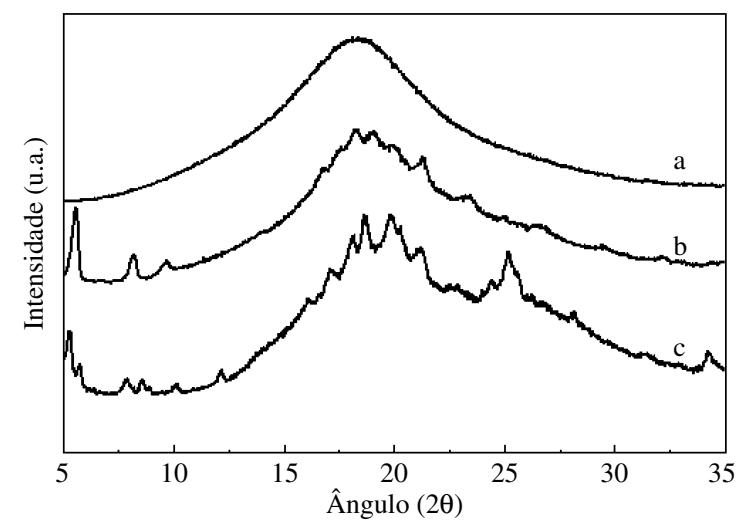

Figura 5. Difratogramas de raios $X$ dos filmes: a) borracha natural; b) compósito B2; e c) PANI-DBSA.

\section{Conclusões}

Compósitos foram obtidos por polimerização em emulsão da anilina na presença de borracha natural e do ácido dodecilbenzeno sulfônico. Condutividade elétrica de aproximadamente 14 ordens de grandeza maior do que a borracha foi obtida para o compósito. Verificou-se que houve formação da polianilina dopada com DBSA nos compósitos, sendo ela a responsável pelo alto valor de condutividade. Não houve variação na transição vítrea do compósito em função do conteúdo de PANI-DBSA, indicando a imiscibilidade dos polímeros. Verificou-se também que a borracha não altera significantemente a fase cristalina da PANI-DBSA no compósito.

\section{Referências Bibliográficas}

1. Kang, E. T.; Neoh, K. G. \& Tan, K. L. - Prog. Polym. Sci., 23, p.211 (1998).

2. Österholm, J. E.; Cao, Y.; Klavetter, F. \& Smith, P. Polymer, 35, p.2902 (1994).

3. Barra, G. M. O.; Jacques, L. B.; Oréfice, R. L. \& Caneiro, J. R. G. - E. Pol. J., 40, p.2017 (2004).

4. Rao, P. S.; Subrahmanya, S. \& Sathyanarayana, D. N. - Synth Met., 143, p.323 (2004).

5. Pud, A.; Ogurtsov, N.; Korzhenko, A. \& Shapoval, G. Prog. Polym. Sci., 28, p.1701 (2003).

6. Steinbüchel, A. - Current Opinion in Microbiology, 6, p.261 (2003).

7. Tanaka, Y. - Rubber Chem. Technol., 64, p.325 (1991).

8. Faez, R. \& De Paoli, M. A. - Journal of Applied Polymer Science, 82, p.1768 (2001).

9. Vallim, M. R.; Felisberti, M. I. \& De Paoli, M. A. - Journal of Applied Polymer Science, 75, p.677 (2000).
10. Yong, K. C; Foot, P. J. S. \& Morgan, H. - European Polylimer Journal, 42, p.1716 (2006).

11. Sreeja, R; Sharma, P. D; Najidha S, Jayan,S. R. \& Predeep, P. - Polymers \& Polymers Composites, 14, p.261 (2006).

12. Camillo, E. C.; Constantino, C. J. L; Teruya, M. Y.; Alves, N.; Mattoso, L. H. C. \& Job, A. E. - Journal of Applied Polymer Science, 97, p.1498 (2005).

13. Schmidt, V.; Domenech, S. C.; Soldi, M. S.; Pinheiro, E.A. \& Soldi, V. - Polymer Degradation and Stability, 83, p.519 (2004).

14. Soares, B. G.; Amorim, G. S.; Souza, F. G.; Oliveira, M. G. \& da Silva, J. E. P. - Synth. Met. 156, p.91 (2006).

15. Leyva, M. E.; Barra, G. M. O. \& Soares, B. G.- Synth. Met., 123, p.443 (2001).

16. Smits, F. M. - The bell System Tech. J., 20, p.711 (1958).

17. Girotto, E. M. \& Santos, I. A. - Química Nova, J 25, p.639 (2002).

18. Han, M. G.; Cho, S. K. \& Oh, S. G. - Synth. Met., 126, p.53 (2002).

19. Han, D.; Chu, Y. ; yYang, L. \& Liu, Y. - Colloids and Surfaces A: Physicochem. Eng. Aspects, 259, p.179 (2005).

20. Lu, X.; Yen Ng, H.; Xu, J. \& He, C. - Synth. Met. 128, p.167 (2002).

21. Yong, K. C.; Foot, P. J. S.; Morgan, H.; Cook, S. \& Tinker A. J. - European Polymer Journal, 42, p.1716 (2006).

22. Rippel, M. M.; Lee, L. T.; Leite, C. A. P. \& Galembeck, F. - J. Colloid Interface Sci., 268 (2), p.330 (2003).

23. Healey, A. M., Hendra, P. J. \& West, Y. D. - Polymer, 37, p. 4009 (1996).

24. Kuramoto, N. \& Geniés, E. M. - Synth. Met., 68, p.191 (1995).

25. Haba, Y.; Segal, E.; Narkis, M.; Titelman, G. I. \& Siegmann, A.; - Synt. Met., 106, p.59 (1999).

26. Laska, J. - Journal of Molecular Structure, 701, p.13 (2004).

27. Masters, J. G.; Sun, Y. \& Macdiarmid, A.G. - Synth. Met., 41-43, p.715 (1991).

28. Xia, Y.; Wiesinger, J. W. \& MacDiarmid, A. G. - Chemi. Mater., 7, p.443 (1995).

Enviado: 02/02/06

Reenviado: 12/02/07

Aceito: $16 / 02 / 07$ 